EPJ Web of Conferences 47, 09004 (2013)

DOI: $10.1051 /$ epjconf/20134709004

(C) Owned by the authors, published by EDP Sciences, 2013

\title{
K-band spectroscopic metallicities and temperatures of M-dwarf stars
}

\author{
Bárbara Rojas-Ayala ${ }^{a}$
}

Department of Astrophysics, American Museum of Natural History, Central Park West at 79th Street, New York, NY 10024, USA

\begin{abstract}
I present the metallicity and effective temperature techniques developed for $\mathrm{M}$ dwarf stars by Rojas-Ayala et al. (2010, 2012). These techniques are based on absorption features present in the modest resolution K-band spectra ( $R \sim 2700$ ) of $\mathrm{M}$ dwarfs and have been calibrated using FGK+M dwarf pairs and synthetic atmosphere models. The $\mathrm{H}_{2} \mathrm{O}-\mathrm{K} 2$ index seems to overestimate the effective temperatures of $\mathrm{M}$ dwarfs when compared to interferometric measurements. The metallicity distribution of the M dwarf host candidates by the Kepler Mission hints that jovian-size planets form preferentially around solar and supersolar metallicity environments, while small rocky planet host exhibit a wide range of metallicities, just like in their solar-type counterparts.
\end{abstract}

\section{INTRODUCTION}

M-dwarf stars hold the key to understand the formation of planets, stars and the Milky Way. They are the most numerous stars in the Galaxy, their lifetimes are longer than the Hubble time, and they are the hosts of some of the most interesting planetary systems discovered to date. However, due to their intrinsic faintness and complex spectra, their fundamental properties have been hard to unravel. Here, I present the metallicity and effective temperature techniques based on the strength of the Ca I triplet and the $\mathrm{Na}$ I doublet, and water absorption present in the K-band spectra of M dwarfs developed by Rojas-Ayala et al. [1, 2].

\section{K-BAND EFFECTIVE TEMPERATURES}

The infrared spectrum of $\mathrm{M}$ dwarfs is predominately dominated by water opacity. The depressions due to water absorption from $2.07 \mu \mathrm{m}$ to $2.38 \mu \mathrm{m}$ increase with decreasing spectral type deforming the overall shape of the spectra of $\mathrm{M}$ dwarfs. Rojas-Ayala et al. [2] defined a water index, the $\mathrm{H}_{2} \mathrm{O}-\mathrm{K} 2$ index, which measures the deformation of the spectra due to water absorption using portions of the K-band spectra of $\mathrm{M}$ dwarfs almost free of atomic features. Smaller values of the $\mathrm{H}_{2} \mathrm{O}-\mathrm{K} 2$ index correspond to greater amounts of $\mathrm{H}_{2} \mathrm{O}$ opacity. Rojas-Ayala et al.[2] calibrated the $\mathrm{H}_{2} \mathrm{O}-\mathrm{K} 2$ index as an effective temperature indicator using the BT-Settl-2010 spectral models [3], which is metallicity and gravity insensitive for $3000<T_{\text {eff }}<3800 \mathrm{~K}$.

Ten of the M dwarfs in [2] have interferometric angular diameters by Boyajian et al. [4]. Boyajian et al. [4] used their trigonometric parallax values and literature photometry to calculate their bolometric fluxes and physical diameters, which allows the determination of their effective temperatures as well. For half of these sample, the agreement between the effective temperatures estimated by the $\mathrm{H}_{2} \mathrm{O}-\mathrm{K} 2$

\footnotetext{
ae-mail: babs@amnh.org
}

This is an Open Access article distributed under the terms of the Creative Commons Attribution License 2.0, which permits unrestricted use, distribution, and reproduction in any medium, provided the original work is properly cited. 


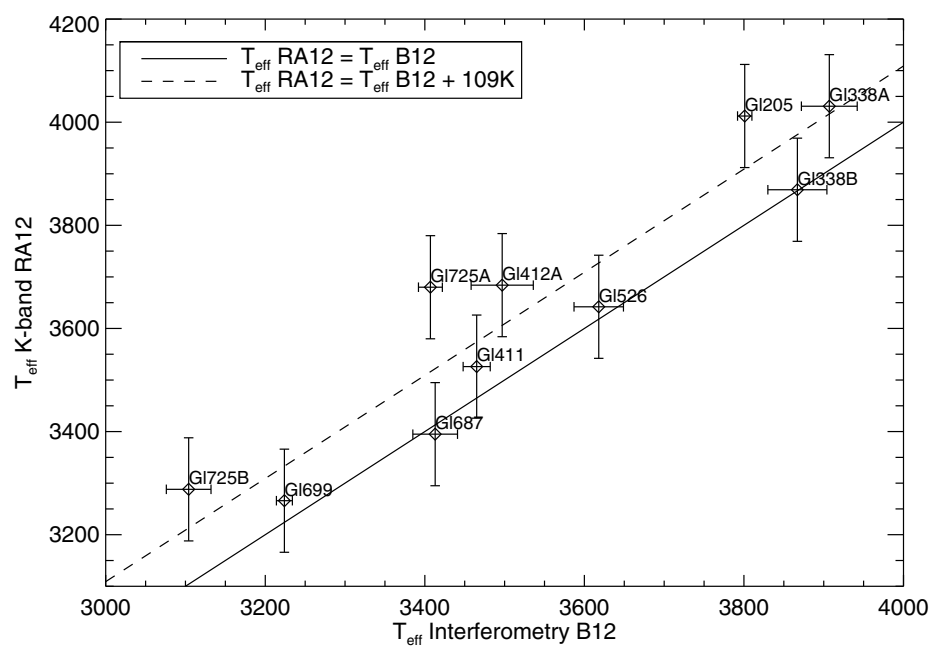

Figure 1. M dwarfs with interferometric measurements by Boyajian et al. (2012) in Rojas-Ayala et al (2012). The $\mathrm{H}_{2} \mathrm{O}-\mathrm{K} 2$ technique overestimates the interferometric temperatures in average by $\sim 109 \mathrm{~K}$.

and interferometric techniques is quite good, with the $\mathrm{H}_{2} \mathrm{O}-\mathrm{K} 2$ technique providing slightly higher $T_{\text {eff }}$ for the stars $\left(\Delta T_{\text {eff }}<61 \mathrm{~K}\right)$. However, for the other half of the sample, the disagreement is higher than $120 \mathrm{~K}$, with Gl 724A having the largest difference: $\Delta T_{\text {eff }}=276 \mathrm{~K}$. If all the sample is considered, the $\mathrm{H}_{2} \mathrm{O}-\mathrm{K} 2$ technique overestimates the interferometric temperatures in average by $\sim 109 \mathrm{~K}$ (Fig. 1).

\section{K-BAND METALLICITIES}

Using FGK + M binary systems, Rojas-Ayala et al. [1, 2] demonstrated that the strength of the Ca I triplet and the $\mathrm{Na}$ I doublet, and water absorption in the K-band differentiate metal-rich and metal-poor M-dwarf stars. The strength of this technique is that it only requires K-band modest resolution $(\sim 2700)$ spectra to provide metallicities with $\sigma([\mathrm{M} / \mathrm{H}]) \sim 0.1$ dex. Using this technique, Rojas-Ayala et al. [2] estimated the metallicities of 133 nearby $\mathrm{M}$ dwarf stars and confirmed that the ground-based discovered jovian M-dwarf planet hosts were more metal-rich than neptune and super Earth M-dwarf planet hosts, which is in agreement with the metallicity distribution of their FGK counterparts.

The K-band $[\mathrm{M} / \mathrm{H}]$ technique was applied to cool stars in the 2011 Kepler Object of Interest (KOI) list [5], by Muirhead et al. [6]. The sample was observed with the same instrument used to develop the metallicity technique [7]. Muirhead et al. [6] estimated the stellar radii of the host candidates and the sizes of their planet candidates using their K-band $[\mathrm{M} / \mathrm{H}]$ and $T_{\text {eff }}$ values and evolutionary models [8],. The distribution of the metallicities of the KOI M-dwarf host candidates is shown in Fig. 2. The sample was divided according to the largest planetary candidate in the systems. The few $\mathrm{M}$ dwarfs with planets larger than $5.5 \mathrm{R}_{\oplus}$ ("Jupiters") exhibit $[\mathrm{M} / \mathrm{H}]$ values higher than $-0.13 \mathrm{dex}$, while the systems with planets smaller than $5.5 \mathrm{R}_{\oplus}$ ("Neptunes" and "Earths") only, exhibit a wide range of metallicities from -0.37 dex to +0.4 dex. This result is in agreement with the one found by Buchhave et al. [9] for a subsample of solar-type stars in the KOI sample.

\section{DISCUSSION AND FUTURE WORK}

The determination of $\mathrm{M}$ dwarf metallicities and temperatures is relevant to stellar astrophysics and planetary formation. The K-band techniques by Rojas-Ayala et al. [1, 2] have been useful to constrain the physical parameters of $\mathrm{M}$ dwarf stars and their planetary systems [6, 10], and provide an alternative 


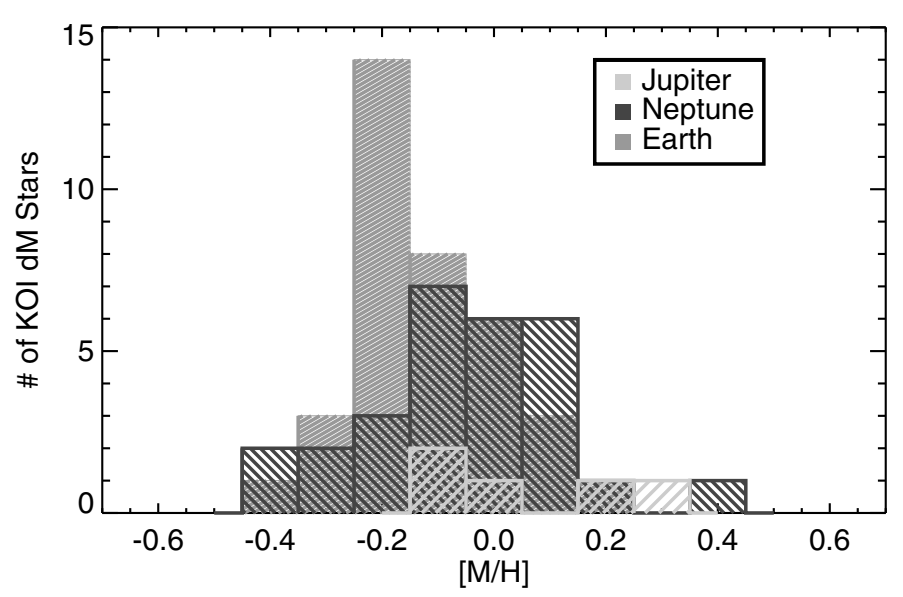

Figure 2. Metallicity distribution of $M$ dwarf planet host candidates in the KOI sample. The sample was divided according to the largest planet candidate in the system. The median values for the distribution of jupiter, neptune, and earth host candidates are $+0.02,-0.05$, and -0.12 dex, respectively.

to optical techniques. During 2012, three other spectroscopic techniques to estimate the metallicities of $\mathrm{M}$ dwarfs have been developed using the near infrared wavelengths [11-13], along with two optical techniques $[14,15]$. Two of these techniques have confirmed that the K-band $\mathrm{Na}$ I doublet and the $\mathrm{Ca}$ I triplet seem to be the best features for the estimation of $\mathrm{M}$ dwarf metallicities in the near infrared at modest resolution $[12,13]$.

Longer wavelengths can also provide metallicity information for $\mathrm{M}$ dwarfs. A new technique based on a color-color diagram, including WISE magnitudes, can produce $[\mathrm{Fe} / \mathrm{H}]$ estimates for $\mathrm{M}$ dwarfs accurate to RMSE $=0.17 \mathrm{dex}$, without the need of parallaxes like previous photometric calibrations (Rojas-Ayala et al. in prep [16]).

\section{References}

[1] B. Rojas-Ayala, K.R. Covey, P.S. Muirhead, J.P. Lloyd, ApJL 720, L113 (2010)

[2] B. Rojas-Ayala, K.R. Covey, P.S. Muirhead, J.P. Lloyd, ApJ 748, 93 (2012), 1112. 4567

[3] F. Allard, D. Homeier, B. Freytag, ArXiv e-prints (2010), 1011.5405

[4] T.S. Boyajian, K. von Braun, G. van Belle, H.A. McAlister, T.A. ten Brummelaar, S.R. Kane, P.S. Muirhead, J. Jones, R. White, G. Schaefer et al., ApJ 757, 112 (2012), 1208. 2431

[5] W.J. Borucki, D.G. Koch, G. Basri, N. Batalha, A. Boss, T.M. Brown, D. Caldwell, J. ChristensenDalsgaard, W.D. Cochran, E. DeVore et al., ApJ 728, 117 (2011)

[6] P.S. Muirhead, K. Hamren, E. Schlawin, B. Rojas-Ayala, K.R. Covey, J.P. Lloyd, ApJL 750, L37 (2012), 1109. 1819

[7] T.L. Herter, C.P. Henderson, J.C. Wilson, K.Y. Matthews, G. Rahmer, M. Bonati, P.S. Muirhead, J.D. Adams, J.P. Lloyd, M.F. Skrutskie et al., The performance of TripleSpec at Palomar, in Society of Photo-Optical Instrumentation Engineers (SPIE) Conference Series (2008), Vol. 7014 of Presented at the Society of Photo-Optical Instrumentation Engineers (SPIE) Conference

[8] A. Dotter, B. Chaboyer, D. Jevremović, V. Kostov, E. Baron, J.W. Ferguson, ApJS 178, 89 (2008), 0804.4473

[9] L.A. Buchhave, D.W. Latham, A. Johansen, M. Bizzarro, G. Torres, J.F. Rowe, N.M. Batalha, W.J. Borucki, E. Brugamyer, C. Caldwell et al., Nature 486, 375 (2012)

[10] G. Anglada-Escudé, B. Rojas-Ayala, A.P. Boss, A.J. Weinberger, J.P. Lloyd, ArXiv e-prints (2012), 1210.8087 
[11] A. Önehag, U. Heiter, B. Gustafsson, N. Piskunov, B. Plez, A. Reiners, A\&A 542, A33 (2012), 1112.0141

[12] R.C. Terrien, S. Mahadevan, C.F. Bender, R. Deshpande, L.W. Ramsey, J.J. Bochanski, ApJ 747, L38 (2012), 1202.1800

[13] A.W. Mann, J.M. Brewer, E. Gaidos, S. Lepine, E.J. Hilton, ArXiv e-prints (2012), 1211. 4630

[14] V. Neves, X. Bonfils, N.C. Santos, X. Delfosse, T. Forveille, F. Allard, C. Natário, C.S. Fernandes, S. Udry, A\&A 538, A25 (2012), 1110. 2694

[15] V. Neves, X. Bonfils, N.C. Santos, X. Delfosse, T. Forveille, F. Allard, S. Udry, ArXiv e-prints (2012), 1212.3372

[16] B. Rojas-Ayala, in preparation (2012) 